\title{
QUALITY ASSESSMENT OF PROCESS MODELS IN PROCESS MINING: THE CASE OF PETRI NETS
}

\author{
Cristina-Claudia OSMAN \\ Business Informatics Research Center, Babeș-Bolyai University Cluj-Napoca \\ cristina.osman@econ.ubbcluj.ro
}

\begin{abstract}
Nowadays, information systems produce and consume enormous quantities of data. The data produced by Process Aware Information Systems is stored as event logs and can be converted into visual representations by using Process Mining algorithms. This paper follows a case study design with in-depth analysis of discovered Petri Nets' quality. First, the theoretical dimensions of the research concerning the quality assessment of process models are presented, followed by a case study describing an electronic invoicing process. The process is analyzed using discovery Process Mining algorithms having as output Petri Nets. The results of four Process Mining algorithms are analyzed and qualitatively evaluated. Most process discovery algorithms consider only Fitness as qualitative criteria. Our study considers both Fitness and Precision metrics in the evaluation of discovered Petri Nets' quality. The overall metric is also discussed. The findings of this study show similar percentages of Fitness and Precision for 3 out of 4 analyzed models, the best overall percentage being $90 \%$.
\end{abstract}

Keywords: quality of process models, Petri Net, process mining, fitness, precision

JEL classification: C55, M15, M21

DOI: $10.12948 / \mathrm{ie} 2019.04 .09$

\section{Introduction}

In the context of Digital Era operational business processes are supported by information systems. Subsequently, a lot of data about user-computer interaction is generated. A ProcessAware Information System (PAIS) is defined as a software system that manages and executes operational processes involving people, applications, and/or information sources on the basis of process models [1]. Workflow Management Systems (WfMSs), Customer-Relationship Management Systems (CRMSs), Enterprise Resource Planning Systems (EPRs), and Business Process Management Systems (BPMSs) are only some examples of PAISs. There is a weighty difference between WfMSs and BPMSs as the last ones also support the diagnosis phase [2]. One of the most significant feature of PAISs is given by their capability to automatically generate event logs. Howbeit, there also are approaches that offer solutions for systems that are not automatically generating event logs [3],[4],[5]. Recently, an extension of PAISs was proposed, namely PAIS-DQ (Data Quality) [6]. Besides classical layers of a PAIS, as they were defined in [1]: Presentation, Process, Application and Persistence, a Data Quality layer was introduced in [6]. The last one applies only on the first three standard layers of a PAIS.

Event logs represent the starting of Process Mining [7]. The key aspects of Process Mining are based on Machine Learning methods combined with Business Process Management techniques. Most Process Mining approaches focus on the conversion of event logs into process models by using different algorithms. But discovery of diagrammatic models is not the only type supported by Process Mining, there also exist conformance checking algorithms designed to compare the event log to the discovered model, while the last type supported by Process Mining points out to enrich the semantics of discovered models. 
One of the oldest modelling languages used in process modelling is represented by Petri Nets [8]. The semantics of Petri Nets is simple and the diagrammatic visualization consists of a bipartite graph comprising transitions and places. Afterwards, other modelling languages like Event-driven Process Chain (EPC), UML Activity Diagrams and BPMN diagrams emerged. A top-down quality framework that proposes process model quality features is SEQUAL [9]. The quality levels considered by SEQUAL Framework are based on Stamper's Semiotic Ladder [10]: physical, empirical, syntactic, semantic, pragmatic, and social. QoMo (Quality of Modelling) is another quality framework based on SEQUAL where several goals for modelling are defined (for instance product goals, usage goals, creation goals, validation goals and so on). In the same vein as PAISs have layers like Data Quality, process models' quality may be quantified by using the frameworks remained earlier, the quality of process models discovered from event logs is also evaluated using metrics like: Fitness, Precision, Generalization and Simplicity [12],[13].

Frequently, the quality of a discovered process model is measured only based on how the process model can reproduce the behavior seen in the event log. This metric is called Fitness. Precision measures the behavior seen in the process model, but it is not in the event log. The ability to replicate future behavior of the process model is called generalization, while simplicity refers to lasagna processes and how human readable they are [14].

This paper discusses a case study of Petri Nets evaluation. The event log used to compute the metrics describes an electronic invoicing process [15]. The quality of the discovered models is measured using conformance Process Mining algorithms.

The remaining part of the paper proceeds as follows. Section 2 presents the event log summary. This section explores basic information like the number of traces, resources or events. Moreover, a general process model extracted from the event log is also depicted. A Process Mining algorithm focused on frequent behavior is used. Section 3 introduces the discovery analysis approach as a comparison of the resulted process models together with the data from event log summary. Section 4 is concerned on the assessment of process models from Fitness and Precision perspectives. The overall metric is also evaluated. Finally, the conclusion section gives a brief summary and critique of the findings.

\section{Event log summary}

The event log used for the comparison of control-flow algorithms describes an electronic invoicing process and the tool used for the current study is ProM Framework [16]. In our analysis we considered only complete events. The event log consists of 20135 cases and 134383 events executed by 6 types of resources (from group 1 to group 6). The process hidden behind the event log takes more than one month.

There are 7 activities mapped on the events: Approve Invoice, Liquidation, Invoice Scanning, Scanning of Extra Documentation, Approve Liquidated Invoices, Marking Paid Invoices, and Payment Approval. Each trace starts with Invoice Scanning activity and at the end of the process, the invoices are either marked as paid (Marking Paid Invoices activity), or approved (Approve Invoice activity). The minimum number of events from a case is 3 , while the maximum number is 9. More than half of the cases (10726) follow the same pattern consisting of 7 activities: Invoice Scanning, Scanning of Extra Documentation, Approve Invoice, Liquidation, Approve Liquidated Invoices, Payment Approval and Marking Paid Invoices. The resources from group 1 perform almost $30 \%$ of activities from the entire process.

The event log describes four types of possible variants. Each variant describes a simple process model: two of the variants generate sequential process models, while the other two provide sequential process models embedded with loops. The figure bellow depicts the behavior of each variant in terms of Heuristics Nets [17]. Although, the algorithm removes infrequent 
behavior and is not able to relate routing sequences like decision or parallelism, loops are properly identified.

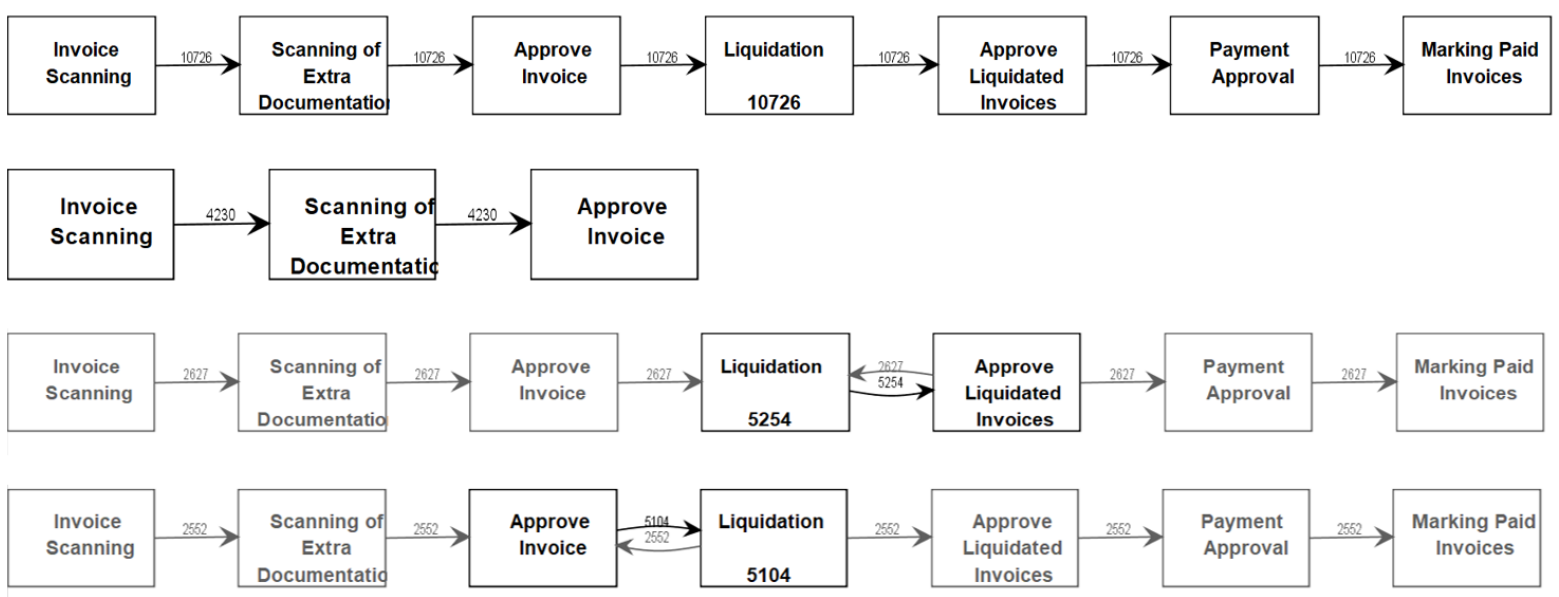

Figure 1. Heuristics Nets of event log

The Dotted Chart illustrates that resources work from Monday to Friday. Moreover, each type of resource executes specific activities, for example the resources belonging to group 2 execute activity Approve invoice, while resources from group 1 execute scanning activities like Invoice Scanning or Scanning of Extra Documentation.

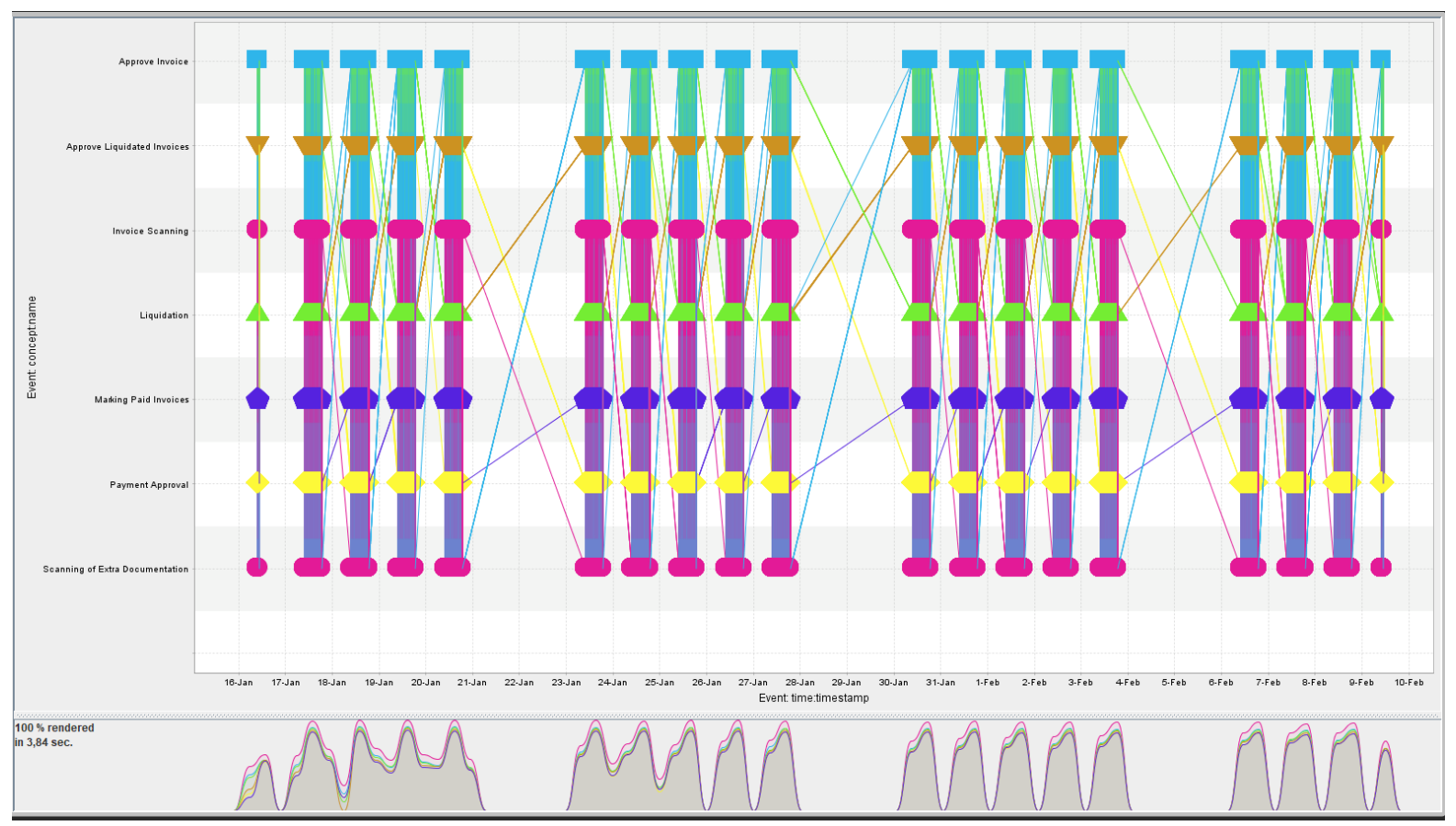

Figure 2. Dotted Chart applied on the event log

Most control-flow algorithms (for instance Alpha Miner [18], Heuristics Miner [17], Evolutionary Tree Miner [12], [13]) use the Causal Activity Matrix in process models extraction. 
www.conferenceie.ase.ro

\begin{tabular}{|c|c|c|c|c|c|c|c|}
\hline From $\backslash$ To & $\begin{array}{c}\text { Approve } \\
\text { Invoice+start }\end{array}$ & $\begin{array}{l}\text { Approve Liquidated } \\
\text { Invoices+start }\end{array}$ & $\begin{array}{c}\text { Invoice } \\
\text { Scanning+start }\end{array}$ & Liquidation+start & $\begin{array}{l}\text { Marking Paid } \\
\text { Invoices+start }\end{array}$ & $\begin{array}{c}\text { Payment } \\
\text { Approval+start }\end{array}$ & $\begin{array}{l}\text { Scanning of Extra } \\
\text { Documentation+start }\end{array}$ \\
\hline Approve Invoice+start & 0.0 & 0.0 & 0.0 & 0.7570204664445502 & 0.0 & 0.0 & -0.9999503377036154 \\
\hline $\begin{array}{l}\text { Approve Liquidated } \\
\text { Invoices+start }\end{array}$ & 0.0 & 0.0 & 0.0 & -0.7516540642722117 & 0.0 & 0.9999371306425249 & 0.0 \\
\hline $\begin{array}{c}\text { Invoice } \\
\text { Scanning+start }\end{array}$ & 0.0 & 0.0 & 0.0 & 0.0 & 0.0 & 0.0 & 0.9999503377036154 \\
\hline Liquidation+start & -0.7570204664445502 & 0.7516540642722117 & 0.0 & 0.0 & 0.0 & 0.0 & 0.0 \\
\hline $\begin{array}{l}\text { Marking Paid } \\
\text { Invoices+start }\end{array}$ & 0.0 & 0.0 & 0.0 & 0.0 & 0.0 & -0.9999371306425249 & 0.0 \\
\hline $\begin{array}{c}\text { Payment } \\
\text { Approval+start }\end{array}$ & 0.0 & -0.9999371306425249 & 0.0 & 0.0 & 0.9999371306425249 & 0.0 & 0.0 \\
\hline $\begin{array}{l}\text { Scanning of Extra } \\
\text { Documentation+start }\end{array}$ & 0.9999503377036154 & 0.0 & -0.9999503377036154 & 0.0 & 0.0 & 0.0 & 0.0 \\
\hline
\end{tabular}

Figure 3. Causal Activity Matrix of event log based on Directly Follows miner

Figure 3 presents the corresponding Causal Activity Matrix of the event log. For instance, the matrix shows a directly-follows relation between Approve Invoice and Liquidation activities.

\section{Discovery analysis}

Discovery analysis represents the comparison of the resulted process models with the data from event log summary. We focus on the most frequent behavior. The Heuristics Net showing the most frequent paths was described in the previous section in order to offer an overview of the process. Next, we will describe the algorithms used in our analysis.

Alpha Miner [18] is the first Process Mining algorithm. The basic idea of Alpha Miner algorithm is to provide visual representations in terms of Petri Nets. Throughout time, several versions of this algorithm have been developed: Alpha+ [19], Alpha++ [20] and Alpha\# [21]. Being the first Process Mining algorithm, it has a series of drawbacks as the incapacity of discover short loops, invisible tasks or duplicated tasks, but Alpha+ and Alpha++ solve these problems.
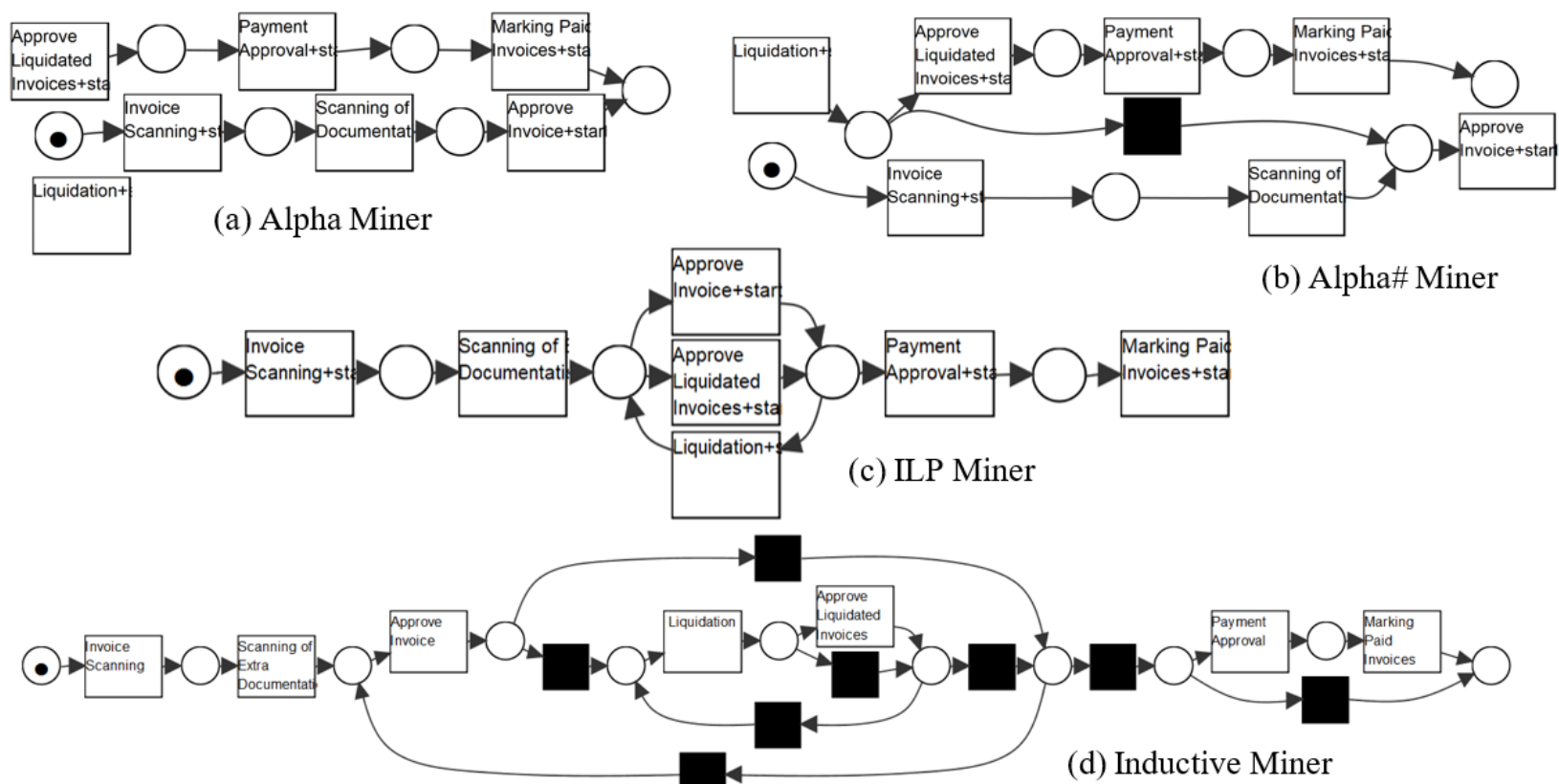

Figure 4. Petri Net discovery using different algorithms

Other Process Mining algorithms providing a Petri Net as result are Inductive [22] and ILP (Integer Liner Programming) Miner [23]. Hereinafter, a series of Petri Net models are 
generated based on the event log. The employed algorithms are: Alpha Miner, Alpha\# Miner, Inductive Miner, respectively ILP Miner. The resulted Petri Nets are depicted in Figure 4. Alpha Miner discovers a Petri Net where transitions are displayed sequentially. Furthermore, Liquidation transition is not linked to any place. Alpha\# Miner seems to provide a more reliable Petri Net. Both models provided by Alpha and Alpha\# Miners are unsound. The Petri Net generated using ILP Miner is sound, but it has missing behavior (for example according to the resulted Petri Net, all traces end with Marking Paid Invoices transition).

Inductive Miner also proposes a sound Petri Net. Both, Inductive and Alpha\# Miners use silent transitions in process discovery. However, Inductive Miner is able to discover two possible end transitions as the summary of event log indicated: Marking Paid Invoices or Approve Invoice. But it also includes one more end activity: Approve Liquidated Invoices. Consequently, the model is overfitting. At a first glance, Inductive Miner is the algorithm that provides the optimal Petri Net by comparing the data from event log summary to the resulted Petri Net. Next section presents the evaluation of algorithms from quality perspective of discovered process models.

\section{Evaluation of algorithms}

This section describes the approach used in the evaluation of Process Mining algorithms. The analysis is performed on algorithms whose output is a Petri Net. Although, Prom Framework supports a series of control-flow oriented algorithms, Fitness and Precision percentages are not computed for all of them. Consequently, the evaluated algorithms are: Alpha Miner, Alpha\# Miner, Inductive Miner, respectively ILP Miner.

The quality of each discovered process model is summarized in Table 1. The Petri Net discovered using Inductive Miner guarantees a Fitness of 95\%, Fitness being the metric which guarantees the mapping of every trace to the Petri Net. The lowest Fitness degree belongs to the Petri Net provided by Alpha\# Miner (68\%).

Table 1. Evaluation metrics for control-flow algorithms

\begin{tabular}{|l|c|c|c|}
\hline \multicolumn{1}{|c|}{ Algorithm } & Fitness & Precision & Overall \\
\hline Alpha Miner & 0.75 & 0.37 & 0.56 \\
\hline Alpha\# Miner & 0.68 & 0.56 & 0.62 \\
\hline ILP Miner & 0.71 & $\mathbf{0 . 7 5}$ & 0.73 \\
\hline Inductive Miner & $\mathbf{0 . 9 5}$ & 0.85 & $\mathbf{0 . 9 0}$ \\
\hline
\end{tabular}

Nevertheless, the Precision computed for the Petri Net generated by Inductive Miner is lower than the Fitness metric because the Petri Net also allows the execution of traces that are not depicted by the event log (for example, according to the model, a trace may end with Approve Liquidated Invoices transition, but the event log describes only Marking Paid Invoices or Approve Invoice as ending transitions). The greatest Precision percentage belongs to ILP Miner, while the lowest Precision percentage is computed for Alpha Miner. The overall evaluation shows that Inductive Miner provides the best Petri Net with an overall metric of $90 \%$.

\section{Conclusions}

This case study seeks to examine the quality of process discovery Process Mining algorithms having as output a Petri Net. Fitness and Precision are computed for the Petri Nets generated 
www.conferenceie.ase.ro

by four Process Mining algorithms: Alpha Miner, Alpha\# Miner, Inductive Miner, respectively ILP Miner. The overall metric is also computed.

Most process discovery algorithms consider only Fitness as qualitative criteria. Undeniably, the results of this study show that the model having the greatest Fitness (95\%), also measures the greatest Precision (85\%), specifically the Petri Net discovered by Inductive Miner. Nevertheless, there are Process Mining algorithms quantified with a considerable Fitness metric, but the Precision degree is not weighty (for instance Alpha Miner). On the other hand, Inductive Miner and ILP Miner provide similar values for both metrics $(71 \%$ versus $75 \%$, respectively $95 \%$ versus $85 \%$ ).

This study may be extended by evaluating other types of models, for instance Process Trees, by including all four dimensions that evaluate the quality of discovered process models.

\section{References}

[1] M. Dumas, W.M.P van der Aalst, A. H. M ter Hofstede, "Process-Aware Information Systems: Bridging People and Software through Process Technology," Chichester: WileyInterscience, 2005, pp. 3-20.

[2] W. M. P. van der Aalst, A. H. M. ter Hofstede, M. Weske, "Business Process Management: A Survey", in: van der Aalst W.M.P., Weske M. (eds) Business Process Management. BPM 2003. Lecture Notes in Computer Science, vol. 2678. Springer, Berlin, Heidelberg, pp. 1-12.

[3] R. Perez-Castillo, B. Weber, J. Pinggera, S. Zugal, I.G.R., de Guzman, M. Piattini, "Generating event logs from non-process-aware systems enabling business Process Mining”, Enterprise Information Systems, vol. 5, no. 3, 2011, pp. 301-335.

[4] C. C. Osman, "SQL Mining: Knowledge Discovery from DML Statements", in Proceedings of the 28th International Business Information Management Association, Seville, Spain, 2016.

[5] W.M. van der Aalst, "Extracting event data from databases to unleash Process Mining", in BPM-Driving innovation in a digital world Springer, Cham. 2015, pp. 105-128.

[6] L. Parody, M. T. López, I. Bermejo, I. Caballero, R.M. Gasca, M. Piattini, "PAIS-DQ: Extending process-aware information systems to support data quality in PAIS life-cycle" in IEEE Tenth International Conference on Research Challenges in Information Science Grenoble, France, 2016, pp. 1-12.

[7] W. van der Aalst, "Process Mining: Data science in action," Springer, Berlin, Heidelberg, 2016.

[8] C. A. Petri. "Kommunikation mit Automaten," PhD thesis, Institut fur instrumentelle Mathematik, Bonn, Germany, 1962.

[9] J. Krogstie, G. Sindre, and H. Jorgensen, "Process models representing know ledge for action: a revised quality framework", in European Journal of Information Systems, vol. 15, no. 1, 2006, pp. 91-102.

[10] R. Stamper, "Signs, norms, and information systems", in Proceedings of the Joint ICL/University of Newcastle Seminar on the Teaching of Computer Science, Part IX: Information, Berlin, Germany, 1996, pp. 1-35.

[11] P. van Bommel, S.J.B.A. Hoppenbrouwers, H.A. Proper, "QoMo: A Modeling Process Quality Framework Based on Sequal”, in: Proceedings of EMMSAD 2007, Trondheim, Norway. 2007, pp. 118-127.

[12] J. C. Buijs, B. F. Van Dongen, W. M. van der Aalst, "On the role of fitness, precision, generalization and simplicity in process discovery", in OTM Confederated International Conferences "On the Move to Meaningful Internet Systems", Rome, Italy, 2012, pp. 305 322. 
[13] J. C. Buijs, B. F. Van Dongen, W. M. van der Aalst, "Quality dimensions in process discovery: The importance of fitness, precision, generalization and simplicity", in International Journal of Cooperative Information Systems, vol. 23, no. 1, 2014, pp 1-39.

[14] W. M. van der Aalst, "Process Mining: discovering and improving Spaghetti and Lasagna processes", In IEEE Symposium on Computational Intelligence and Data Mining, Paris, France, 2011, pp. 1-7.

[15] A. Djedović, (2018, June 27), "Electronic Invoicing Event Logs," 4TU. Centre for Research Data, Available at: https://doi.org/10.4121/uuid:5a9039b8-794a-4ccd-a5ef4671f0a258a4 [Accessed: February 15, 2019].

[16] H. M. W. Verbeek, J. C. A. M. Buijs, B. F. van Dongen, W. M. van der Aalst, "Prom 6: The process mining toolkit", in Proceedings of BPM Demonstration Track, vol. 615, 2010, pp. 34-39.

[17] A. J. M. M. Weijters, J. T. S. Ribeiro, "Flexible Heuristics Miner (FHM)", in BETA Working Paper Series, WP 334, Eindhoven University of Technology, Eindhoven, 2010.

[18] W. van der Aalst, T. Weijters, L. Maruster, "Workflow Mining: Discovering Process Models from Event Logs", in IEEE Transactions on Knowledge and Data Engineering, vol. 16, no. 9, 2004, pp. 1128-1142.

[19] A. K. A. de Medeiros, B. F. van Dongen, W.M.P. van der Aalst, A.J.M.M. Weijters, "Process Mining: Extending the alpha-algorithm to mine short loops", in BETA working paper series 113, Eindhoven University of Technology, 2004.

[20] L. Wen, W.M.P. van der Aalst, J. Wang, J. Sun, "Mining process models with non-freechoice constructs", in Data Mining and Knowledge Discovery, vol. 15, no. 2, 2007, pp.145-180.

[21] L. Wen, J. Wang, W.M.P. van der Aalst, B. Huang, J. Sun, "Mining process models with prime invisible tasks", in Data \& Knowledge Engineering, vol. 69, no. 10, 2010, pp. 9991021.

[22] S. J. J. Leemans, D. Fahland, W. M .P. van der Aalst, "Process and deviation exploration with Inductive visual Miner", in CEUR Workshop Proceedings, vol. 1295, Proceedings of the BPM Demo Sessions 2014 Co-located with the 12th International Conference on Business Process Management, Eindhoven, The Netherlands, 2014, pp. 46-50.

[23] J. M. E. M. van der Werf, B.F. van Dongen, C.A.J. Hurkens, A. Serebrenik, "Process Discovery using Integer Linear Programming", in Fundamenta Informaticae, vol. 94, no. 3-4, 2010, pp. 387-412. 